\title{
ORIGINAL
}

\section{EL MODELADO FAMILIAR Y EL PAPEL EDUCATIVO DE LOS PADRES EN LA ETIOLOGÍA DEL CONSUMO DE ALCOHOL EN LOS ADOLESCENTES}

\author{
Javier Pons Diez \\ Área de Psicología Social. Universitat de València.
}

\section{RESUMEN}

Fundamento: Se pretende determinar las relaciones existentes entre el consumo de bebidas alcohólicas por parte de los adolescentes y dos variables psicosociales: el consumo alcohólico de los otros miembros del sistema familiar y las estrategias educativas paternas.

Métodos: Se seleccionó una muestra de 1.100 adolescentes de ambos sexos, de edades comprendidas entre los 15 y los 19 años en la ciudad de Valencia. Se evaluaron a través de los instrumentos pertinentes tres variables: a) estrategias paternas de socialización familiar; b) percepción del adolescente respecto al consumo de siete tipos de bebidas alcohólicas en los miembros de su familia; y c) consumo de siete tipos diferentes de bebidas alcohólicas por parte de los adolescentes en los fines de semana.

Resultados: Una vez aplicado el análisis de varianza, se detectó que el consumo de alcohol en los adolescentes está significativamente relacionado con el consumo de los otros miembros de su familia, y con la utilización por parte de los padres de estrategias educativas basadas principalmente en la reprobación y en la ausencia de comprensión y afecto.

Conclusiones: Se infiere la necesidad de implicar a los padres en los procesos preventivos a fin de crear un ambiente familiar positivo y además ofrecer a los hijos un modelado racional y controlado en el uso familiar de bebidas alcohólicas. La prevención debería incluir a los padres como un agente central de la intervención.

Palabras clave: Adolesencia. Alcohol. Influencia social. Ciencias sociales. Drogas. Infancia. Toxicomınías.

\section{ABSTRACT \\ Family Modeling and the Educational Role of Parents as Regards the Factors Contribution to the Use of Alcohol among Adolescents}

Background: This analysis is aimed at determining the relationships existing between alcoholic beverage consumption on the part of adolescents and two psychosocial variables: the consumption of alcohol on the part of other members of the fannily system and the paternal educational strategies.

Methods: A sample of 1100 adolescents of both sexes was taken, ranging 15 to 19 years of age in the city of Valencia. Three variables were evaluated using the pertinent tools: a) paternal family socializing strategies; b) the adolescent $»$ s feeling about the consumption of seven types of alcoholic beverages by the members of his/her family; and c) consumption of seven different types of alcoholic beverages on the part of the adolescents on weekends.

Results: Following the application of the variance analysis. it was found that the consumption of alcohol among adolescents is significantly related to the consumption thereof on the part of other members of his/her family, and to the use on the part of the parents of educational strategies based mainly on disapproval and on the lack of understanding and affection.

Conclusions: The need is inferred of involving parents in the preventive processes for the purpose of creating a positive family environment and also of offering children a rational, controlled model with regard to the family use of alcoholic beverages. Prevention should include the parents as a central active figure of involvement.

Key Words: Adolescence. Alcohol. Social Influence. Social Studies. Drugs. Early Childhood. Addictions. $\overline{\text { Correspondencia: }}$

Javier Pons Diez

Área de Psicología Social de la Universitat de València.

Av. Blasco Ibáñez, 21. 46010 Valencia.

Tf.: $96-3864036$

\section{INTRODUCCIÓN}

El sistema familiar juega un papel fundamental para explicar la aparición de diferentes conductas desadaptativas en los hijos. Los padres, intencionadamente o no, son la 
influencia más poderosa en la vida de sus hijos $^{1}$. Las de otros contextos sociales -medios de comunicación, grupo de iguales, escuela,...- pasa normalmente por el tamiz. de la familia, que puede tanto amplificar como disminuir sus efectos e influencias, sean estos positivos o negativos. Muy especialmente en el caso de las drogas institucionalizadas, la actitud más o menos crítica de los padres ante ellas, así como sus propias pautas de consumo, pueden desviar o reforzar el efecto de los medios de comunicación o del grupo de iguales como agentes desencadenantes.

Sin desestimar la importancia de la presión grupal, la influencia de la familia resulta ser la variable que con más insistencia se plantea en los trabajos referidos a factores de riesgo ${ }^{2}$. Esta influencia debe ser contemplada desde dos vertientes. En primer lugar, el consumo de bebidas alcohólicas por parte de los padres puede propiciar el consumo las mismas por los hijos. Por otra parte, la existencia de problemas de relación en la familia y sus consecuencias en el clima familiar y en diversas variables individuales de los hijos, es uno de los principales desencadenantes del aumento de la frecuencia del consumo de bebidas alcohólicas.

Indudablemente, la familia es el primer marco de referencia en el que se inicia la socialización y, por lo tanto, la personalidad del individuo. La familia se especializa en la formación de papeles para sus miembros, más que en preparar las condiciones para la libre asunción de su identidad. Además, la familia como socializador primario del niño, enseña principalmente cómo someterse a la sociedad, al tiempo que deposita en éste un elaborado sistema de restricciones y permisiones. La familia lleva a cabo la enseñanza de los controles sociales mediante la administración de premios y culpas, aplicables a las conductas que se ajusten o no a los criterios descritos por la familia y la sociedad ${ }^{3}$.
Puede considerarse la familia como sistema social, advirtiendo que su característica más sobresaliente es la de ser una pequeña unidad formada por un número relativamente restringido de individuos reunidos por lazos íntimos y complejos, basada esencialmente en acuerdos voluntarios entre adultos y donde predominaría una tendencia democrática en las relaciones, pero con una clara delimitación de roles — cada uno cumple su papel social definido: padre, madre, hijo, hija- encontrándose los hijos en el rol de protegidos y guiados, frente a los adultos que son los protectores y guías. Desde esta perspectiva, el adulto modela en buena parte la conducta del hijo.

Es un hecho constatado que el consumo de drogas por los padres propicia el consumo de las mismas o de otras sustancias por los hijos. A este respecto, algunas investigaciones apuntan a que el consumo habitual de alcohol en los progenitores, se encuentra presente en las historias familiares de aproximadamente la mitad de los adictos a opiá$\operatorname{ceos}^{4,5}$.

En el caso del consumo de bebidas alcohólicas, la influencia familiar es, si cabe, más decisiva, dado el carácter institucionalizado de esta sustancia. Es conocido que el Estado español, además de tradicional productor, se encuentra entre los primeros del mundo en cuanto a consumo de alcohol por habitante y año ${ }^{6}$. Conocido es también que este consumo se encuentra vinculado a usos familiares de forma claramente perceptible para el niño desde su ingreso en la familia.

Esta casi imperceptible inducción al consumo a través de la normalidad de unos usos familiares habituales, es en nuestra cultura absolutamente imposible en el caso de las drogas no institucionalizadas, cuya imagen generalmente viene acompañada de una actitud negativa y una posición crítica. Incluso en el caso del tabaco, y pese a ser una droga de consumo altamente extendido, su consumo está habitualmente vetado a los niños. Raramente los padres incitan este uso en sus 
hijos. Suele ser el grupo de amigos el instigador y, normalmente, el consumo deviene «clandestino» y es ocultado a los mayores. Viene a ser una muestra de independencia y un intento de autoafirmación y afiliación, pero con la conciencia de que es una conducta recriminada o no aceptada por los padres.

Esta transmisión cultural del hábito alcohólico presenta un contrapunto, igualmente merecedor de mención, en la posibilidad de encontrar un componente genético que explique esta transmisión familiar, ilustrada en investigaciones llevadas a cabo desde el ámbito biomédico ${ }^{7-9}$. De la misma forma que estos estudios dejan una posibilidad abierta a las influencias ambientales, en justa medida, hemos de reconocer que una comprensión global de la conducta de abuso no puede descartar las influencias genéticas, por más que nuestro interés y planteamiento de trabajo sea de tipo psicosocial. Sin embargo, precisamente por la naturaleza de nuestro punto de vista, no entraremos en más consideraciones, y entenderemos que la simple mención y consideración a esta perspectiva etiológica, es suficiente para los propósitos de nuestra investigación.

Por otro lado, las percepciones de insatisfacción respecto al medio socializador familiar, también han sido señaladas como destacado factor etiológico del consumo abusivo de alcohol en los adolescentes ${ }^{10-12}$.

La familia, en el ejercicio de su función socializadora, crea en el hijo las bases de su identidad y le enseña a apreciarse a sí mismo, es decir, a desarrollar su autoconcepto y su autoestima. En términos generales, los estilos parentales de socialización apoyativos y afectivos, al contrario que los cohercitivos y reprobativos, desarrollarán en los hijos confianza en sí mismos, alta autoestima y capacidad de autocontrol ${ }^{13.14}$. Cuando, con la llegada de la adolescencia, se incremente la importancia del juicio de los iguales para valorarse uno mismo ${ }^{15}$, el adolescente educado en los términos anteriores podrá ser menos vulnerable a la presión grupal, variable que ha sido ampliamente relacionada con el consumo de alcohol ${ }^{16.17}$.

Lo expuesto en los párrafos precedentes nos lleva a plantear una investigación que determine las relaciones existentes entre el consumo de bebidas alcohólicas por parte de los adolescentes, y dos variables derivadas del contexto familiar: el consumo alcohólico de los otros miembros del sistema familiar, y las estrategias educativas paternas.

\section{SUJETOS Y MÉTODOS}

\section{Muestra}

Para realizar esta investigación, se seleccionó una muestra de 1100 adolescentes de ambos sexos y edades comprendidas entre los 15 y 19 años, escolarizados en centros públicos y privados de la ciudad de Valencia. En la distribución por sexos el $46.9 \%$ de la muestra correspondió a varones y el $53.1 \%$ a mujeres.

En función del tipo de centro de escolarización, el $55.4 \%$ cursan sus estudios en centros públicos y el $44.6 \%$ en centros privados.

Finalmente, y en cuanto a la distribución por edades, el mayor porcentaje corresponde a la categoría de 15 años (36.6\%), seguido de la de 16 años $(24.8 \%), 17$ años (19\%), 18 años $(13.1 \%)$ y 19 años $(6.5 \%)$.

\section{Instrumentos de medida}

Con la finalidad de obtener los datos de esta investigación, se utilizaron tres cuestionarios (ver anexo):

- Escala INFLUCOL-92. Instrumento de elaboración propia que se utilizó para obtener los datos acerca del consumo de alcohol en la familia. El cuestionario consta 21 preguntas, en las que se interroga al adolescente sobre su percepción acerca del consumo de siete tipos diferentes de bebidas alcohólicas 
en tres miembros de su familia: padre, madre y hermanos/as mayores. Los ítems aparecen con una escala de respuesta de cuatro pasos: «nada», «poco», «bastante» $\mathrm{y} \ll \mathrm{mu}-$ cho».

- Escala $E M B U$ 89. Se utilizó para obtener los datos referidos a las estrategias educativas familiares. Este instrumento fue elaborado originalmente por Perris et al. ${ }^{18}$, con el fin de determinar el papel que dichas prácticas educativas desempeñaban en la etiología de diversos trastornos psicológicos como fobias y depresiones. En su origen era un instrumento que evaluaba los recuerdos que sujetos adultos con un determinado diagnóstico clínico, tenían de las estrategias de socialización empleadas por sus padres. Los ítems, por lo tanto, estaban formulados en tiempo pasado. Más adelante, se ha venido contrastando repetidamente su validez y fiabilidad en muestras de sujetos normales de diversas culturas ${ }^{19,20}$.

La versión que aquí utilizamos tiene sus antecedentes en las primeras adaptaciones españolas de la escala original ${ }^{21,22}$. Consta de 81 ítems formulados en tiempo presente y con cinco posibilidades de respuesta dispuestas en una escala tipo Likert: «siempre», «muchas veces», «algunas veces», «pocas veces» y «nunca». La estructura factorial que se ha utilizado es la encontrada por Herrero et al. ${ }^{23}$. Estos autores, utilizando una muestra de adolescentes valencianos de características similares a la nuestra, obtuvieron una estructura compuesta por las siguientes dimensiones, referidas a diferentes estrategias educativas de los padres hacia los hijos:

- Sobreprotección (ítems 1, 12, 18, 20, $26^{*}, 46,56^{*}, 69^{*}, 70$ y 73 ): Este factor hace alusión a prácticas educativas basadas en un excesivo control y preocupación de los padres por lo que puedan hacer sus hijos o por lo que pueda ocurrirles, dificultando su libertad para hacer cosas que otros muchachos/as pueden hacer o escoger.
- Comprensión y apoyo (ítems 2, 4, 13, $21,22,27^{*}, 32,33^{*}, 39,40,41,43,47$, $48,54,62,67,74,75,78$ y 81 ): Los ítems de este factor giran en torno a la percepción de afecto, cariño $\mathrm{y}$ apoyo que el adolescente recibe de sus padres, así como a la facilidad para establecer comunicación en el ámbito familiar y a las demostraciones de amor y respeto hacia el hijo/a.

- Castigo (ítems 6, 19, 23, 28, 30, 45, $55,58,59,63,64,65,68,71,76$ y 77 ): Alusivo a prácticas educativas de tipo represivo, en la que se incluyen castigos físicos, críticas y violencia verbal, como respuesta a la conducta del hijo/a.

- Presión hacia el logro (ítems 7, 9, 14, $25,31,34,35,38,42,52$ y 60): Referidos a la presión que los padres ejercen en su hijo/a para que éste obtenga buenos resultados académicos o sociales. Esta presión se traduce también en preocupación por las amistades que el adolescente frecuenta y el continuo énfasis en que el hijo/a oriente sus acciones hacia el éxito, la competividad y el triunfo.

- Rechazo (ítems 10*, 11*, 16, 17, 29, $61,72,79^{*}$ y $\left.80^{*}\right)$ : Refleja la sensación de rechazo que tiene el hijo/a dentro de su ambiente familiar en relación al trato que recibe de sus padres, en comparación a sus hermanos, así como la queja del adolescente por la falta de percepción de afecto hacia él o de un trato injusto y discriminativo respecto a los otros miembros del sistema familiar. Es obvio que esta sensación de rechazo, tal y como queda definida en esta variable, no aparecería en aquellos adolescentes que no tuvieran hermanos.

- Reprobación (ítems 5, 8, 15, 24, 36, $37,44,49,50,51,53,57$ y 66): Esta dimensión viene definida por contenidos semánticos que aluden a la percep- 
ción filial de incomprensión por parte de los padres. Una puntuación alta en este factor implicaría la existencia en el hijo/a de un sentimiento de ser incomprendido y no aceptado integralmente, así como de una cierta dificultad para satisfacer las propias necesidades en el ámbito familiar. Cabe citar que esta dimensión fue denominada originalmente por sus autores «atribución de culpa», aunque, en nuestro caso, hemos considerado utilizar el nombre de «reprobación», por ser más claro en su significado para expresar el contenido de sus ítems.

Los ítems marcados con * se interpretan de manera inversa.

- Escala HABICOL-92. Fue elaborada para medir los hábitos de consumo de alcohol en la muestra utilizada. Se solicita al adolescente una estimación de su consumo en sicte bebidas alcohólicas diferentes, contempladas éstas en sus diferentes formatos comerciales. La escala tiene 17 ítems y está referida al consumo habitual durante un fin de semana normal con los amigos.

A partir de las respuestas a escala $H A B I$ COL-92, se realizó una estimación del consumo alcohólico que permitió agrupar a los sujetos en tres clusters de consumo - abstemios, moderados y excesivos-, mediante el procedimiento de mínima varianza intragrupo y la máxima varianza entre grupos. De esta forma, el $24.5 \%$ de los sujetos fueron asignados al cluster de abstemios, el $56.8 \%$ al de consumidores moderados y el $18.6 \%$ al de consumidores excesivos.

\section{Procedimiento}

Para establecer las relaciones entre las variables fueron realizados análisis de varianza en los que se utilizó como variable independiente el estatus de consumidor de alcohol, categorizado en los tres clusters de consumo extraídos, y como variables dependientes, las siguientes: a) por un lado, las puntuaciones obtenidas en las tres escalas dcl cuestionario INFLUCOL-92 que reflejan tres categorías de consumo - consumo del padre, consumo de la madre y consumo de los hermanos/as mayores-, que son estimaciones que el adolescente hace del consumo en los miembros de su familia; b) por otro lado, la puntuación obtenida por los sujetos en los seis factores que conforman la escala de socialización familiar.

Posteriormente al análisis de varianza, se realiza un test de Tukey para conocer entre qué niveles de la variable independiente aparecen las diferencias significativas detectadas.

\section{RESULTADOS}

En la tabla 1 se muestran los resultados del análisis de varianza realizado para conocer si existen diferencias significativas en el consumo de alcohol entre los miembros de la familia, en función de la pertenencia a cada uno de los tres clusters de consumo que hemos utilizado.

Los resultados apuntados dejan bastante clara la existencia de una fuerte relación entre las dos variables analizadas. Se observa que con un nivel de significación de $\mathrm{p}<.001$, aparecen diferencias significativas en las tres categorías de la variable dependiente: Consumo del padre, consumo de la madre y consumo de los hermanos/as Mayores.

Para determinar entre qué niveles de la variable independiente se dan estas diferencias, se muestran en la tabla 2 los resultados del tcst de Tukey realizado a este efecto. Éste evidencia que, con un nivel de significación alfa de 0,05, existen diferencias significativas entre los tres grupos de consumidores para las tres categorías de la variable dependiente. Así pues, se observa que conforme aumenta la cantidad de alcohol consumida por el adolescente, se incrementa la consumida por sus familiares. 
Tabla 1

Relaciones entre consumo alcohólico en el adolescente (variable independiente) y consumo familiar (variable dependiente)

\begin{tabular}{|lrrrrr|}
\hline Variable dependiente & S.C. & g.l. & M.C. & F & $P$ \\
\hline Consumo del Padre & 9882.096 & 2 & 4941.084 & 31.382 & $<0.001$ \\
Consumo de la Madre & 3351.242 & 2 & 1675.621 & 20.063 & $<0.001$ \\
Consumo de Hermanos mayores & 20222.695 & 2 & 10111.330 & 56.311 & $<0.001$ \\
\hline Método estadístico: Análisis de Varianza & & & & & \\
\hline
\end{tabular}

Leyenda: S.C.: sumas cuadráticas; g.l.: grados de libertad; M.C.: medias cuadráticas; F: valor de F empírica: P: probabilidad.

Tabla 2

Comparación de medias en consumo alcohólico familiar para cada nivel de la variable independiente

\begin{tabular}{|lccc|}
\hline \multirow{2}{*}{ Variable dependiente } & \multicolumn{3}{c|}{ Niveles de la variable independiente } \\
\cline { 2 - 4 } & Abstemios & Moderados & Excesivos \\
\hline Consumo del Padre & $20.381 \mathbf{c}$ & $24.152 \mathbf{b}$ & $29.585 \mathbf{a}$ \\
Consumo de la Madre & $14.452 \mathbf{c}$ & $16.760 \mathbf{b}$ & $19.815 \mathbf{a}$ \\
Consumo de Hermanos mayores & $10.930 \mathbf{c}$ & $16.515 \mathbf{b}$ & $24.102 \mathbf{a}$ \\
\hline Método estadístico: Test de Contraste de Medias de Tukey (alfa $=0.05)$ & \\
\hline
\end{tabular}

Leyenda: $a>b>c$ (diferencias significativas)

Estos resultados sugieren la fuerte influencia que el consumo de alcohol en el ámbito familiar tiene en el que lleva a cabo el propio adolescente, $\mathrm{y}$, además, apuntan la posibilidad de que las las acciones preventivas se focalicen no sólo sobre el adolescente potencialmente consumidor, sino también sobre sus grupos de influencia, especialmente, en el propio ambiente familiar.

En la tabla 3, se contienen los datos relativos al análisis de varianza realizado. Existen diferencias significativas en cinco de los seis factores de socialización familiar: Comprensión y apoyo $(\mathrm{p}<.001)$, castigo $(\mathrm{p}<.001)$, presión hacia el logro $(\mathrm{p}=.006)$, rechazo $(\mathrm{p}<.001)$ y reprobación $(\mathrm{p}<.001)$. No aparecen diferencias significativas en el factor sobreprotección $(\mathrm{p}=.088)$. Para conocer el sentido de estas diferencias, se aplicó el test de contraste de medias de Tukey. En la tabla 4 se exponen sus resultados. Se comprueba que, para un nivel de significación alfa de 0,05 , las diferencias se dan fun- damentalmente entre los consumidores excesivos, por una parte, y los abstemios y moderados, por otra. Así ocurre en los factores castigo, presión hacia el logro y rechazo, en los que el grupo de consumo excesivo puntúa significativamente más alto que los grupos de abstemios y moderados y también, en el factor comprensión y apoyo, en el que los consumidores excesivos obtienen una puntuación significativamente más baja que los otros dos grupos, no detectándose en estos cuatro factores diferencias significativas entre abstemios y moderados. Por otra parte, en el factor reprobación se observan diferencias significativas entre los tres grupos, de forma tal que a medida que aumenta la cantidad de alcohol consumido, se incrementa la puntuación obtenida en este factor. Pese a no detectarse diferencias significativas, cabe señalar que en el factor sobreprotección, es el grupo de consumidores excesivos el que obtiene la puntuación más alta, mientras que la puntuación más baja corresponde al de moderados. 
Tabla 3

Relaciones entre consumo alcohólico en el adolescente (variable independiente) y estrategías de socialización familiar (variable dependiente)

\begin{tabular}{|lrrrrrr|}
\hline Variable dependiente & \multicolumn{1}{c}{ S.C. } & g.l. & M.C. & \multicolumn{1}{c}{$F$} & $P$ \\
\hline Sobreprotección & 186.699 & 2 & 93.350 & 2.433 & 0.088 \\
Comprensión y Apoyo & 3106.174 & 2 & 1553.087 & 8.121 & $<0.001$ \\
Castigo & 1211.070 & 2 & 605.535 & 12.981 & $<0.001$ \\
Presión hacia el Logro & 367.766 & 2 & 183.883 & 5.093 & 0.006 \\
Rechazo & 288.662 & 2 & 144.331 & 10.657 & $<0.001$ \\
Reprobación & 2049.561 & 2 & 1024.781 & 22.713 & $<0.001$ \\
\hline Método estadístico: Análisis de Varianza & & & & & \\
\hline
\end{tabular}

Leverda: S.C.: sumas cuadráticas; g.l.: grados de libertid; M.C.: medias cuadráticas; F: valor de F empírica; P: probabilidad.

Tabla 4

Comparación de medias en estrategias de socialización familiar para cada nivel de la variable independiente

\begin{tabular}{|c|c|c|c|}
\hline \multirow{2}{*}{ Variable dependiente } & \multicolumn{3}{|c|}{ Niveles de la variable independiente } \\
\hline & Abstemios & Moderados & Excesivos \\
\hline Sobreprotección & $27.815=$ & $27.755=$ & $28.829=$ \\
\hline Comprensión y Apoyo & $81.374 a$ & $79.762 \mathbf{a}$ & $76.288 \mathrm{~b}$ \\
\hline Castigo & $23.204 \mathrm{~b}$ & $23.411 \mathbf{b}$ & $26.034 a$ \\
\hline Presión hacia el Logro & $32.696 \mathrm{~b}$ & $33.088 \mathbf{b}$ & $34.395 a$ \\
\hline Rechazo & $23.081 \mathbf{b}$ & $23.286 \mathbf{b}$ & $24.522 \mathrm{a}$ \\
\hline Reprobación & $27.119 \mathfrak{c}$ & $28.365 \mathbf{b}$ & $31.234 \mathrm{a}$ \\
\hline
\end{tabular}

Leyenda: $\mathrm{a}>\mathrm{b}>\mathrm{c}$ (diferencias significativas); $=$ : no hay diferencias significativas

Podemos realizar, conocidos estos resultados, y en función del contenido de los factores los siguientes apuntes: La pertenencia al grupo de consumidores excesivos se encuentra relacionada con puntuaciones relativamente más elevadas en aquellos factores que hacen referencia a estrategias educativas poco respetuosas con el hijo -castigo, presión hacia el logro, rechazo y reprobación-, y más baja, en comparación a los otros grupos, en el factor que recoge las percepciones de afecto y respeto -comprensión y apoyo-. La excesiva presión hacia el logro y la percepción de incomprensión y no aceptación inherente al factor reprobación, pueden significar un desconocimiento - premeditado o inconsciente- por parte de los progenitores, de las necesidades de autoafirmación y autonomía del adolescente. Esto último, aunque de manera más sutil, no encierra menor agresividad, desde la percepción del hijo, que la utilización manifiesta de estrategias de castigo físico y/o verbal - factor castigo)o la existencia de comportamientos paternos que provoquen en el hijo un sentimiento de ser rechazado o discriminado respecto de sus hermanos - factor rechazo-. Por otra parte, un ambicnte familiar dc comprensión y afecto, donde se facilite la comunicación entre sus miembros, aparece como relativamente más característico de aquellos adolescentes abstcmios o consumidores moderados, lo que pone de manifiesto la importancia de un clima familiar positivo en la prevención de conductas desajustadas por parte de los adolescentes. 


\section{DISCUSIÓN}

Los resultados expuestos en las páginas anteriores ponen de manifiesto la existencia de una fuerte relación entre el consumo de alcohol llevado a cabo por el adolescente durante los fines de semana, y las dos variables dependientes utilizadas: el consumo familiar y las estrategias paternas de socialización.

En el caso de la variable consumo familiar, se han detectado en todos los casos diferencias entre los grupos, con un nivel de significación elevado, de manera que los adolescentes que más consumen, pertenecen a familias en las que el consumo alcohólico es habitual. Algunas investigaciones anteriores realizadas en el Estado español analizaron la influencia que el consumo habitual de bebidas alcohólicas en el ámbito familiar tiene en el de los más jóvenes. Así, en los estudios de Cruz Roja Española ${ }^{24}$, Cárdenas ${ }^{25}$ y Martínez, Aguinaga y Varo ${ }^{26}$, se detectó una relación directa entre el consumo de alcohol de los progenitores y el que realizaban sus hijos adolecentes. El efecto de modelado de una práctica habitual en algunas familias es uno de los factores que puede explicar la ingesta abusiva de sus miembros más jóvenes.

A este respecto, el papel del modelado familiar será decisivo en las acciones preventivas. No se trata de que la familia anule su consumo de bebidas alcohólicas ante sus hijos; más bien se trataría de ofrecer modelos de consumo controlado. Esto es especialmente importante, ya que algunos autores han señalado que los adolescentes que beben de manera abusiva no han alcanzado un aprendizaje del uso moderado de esta sustancia, dado que su consumo ocurre fundamentalmente con el grupo de iguales y no con la familia ${ }^{27}$.

Por lo que se refiere a las estrategias de educación familiar, ha sido comprobado que las diferencias significativas han aparecido fundamentalmente, entre aquellos ado- lescentes que consumen alcohol de manera excesiva y aquellos que no lo hacen, sean estos últimos abstemios o consumidores moderados. Las estrategias paternas que denominamos poco respetuosas con el hijo, aparecen más frecuentemente entre los adolescentes que consumen alcohol de manera abusiva, es decir, aquellos que manifiestan una conducta socialmente desajustada. Dicho de otra manera, el clima de insatisfacción respecto al sistema familiar y a la relación con los progenitores, explicado por la alta puntuación en los factores castigo, presión hacia el logro, rechazo y reprobación, y por la baja puntuación en el factor comprensión y apoyo, se encuentra relacionado con un consumo excesivo de bebidas alcohólicas por parte de los adolescentes. Cabe señalar que investigadores como Elzo et al. ${ }^{28} \mathrm{o}$ Berjano $^{29}$ encontraron relaciones entre el consumo de alcohol en los adolescentes y la percepción de dificultades en la relación familiar.

Ya ha sido apuntado en la introducción de este trabajo que las prácticas educativas basadas en la facilidad para establecer comunicación y en la expresión de afecto, apoyo y comprensión, juegan un papel decisivo en el ajuste social y emocional del hijo. El adolescente se siente de esta manera aceptado, valorado y seguro en la relación con sus padres, percepciones éstas que le acompañarán en el desarrollo de sus propias relaciones sociales con el grupo de iguales. Todo lo contrario ocurre con aquel adolescente que ha crecido desarrollando un sentimier to de incomprensión y de falta de aceptación incondicional por parte de sus padres.

El sentimiento de no ser comprendido y aceptado en su propia personalidad es inherente a la puntuación alta en, al menos, los factores reprobación, presión hacia el logro y rechazo, así como a la puntuación baja en el factor comprensión y apoyo. Por otro lado, la explícita expresión de agresividad contenida en el factor castigo, así como la más sutil caracterizada por la utilización premeditada del afecto como medio para influir 
sobre la conducta del hijo, presente, de nuevo, en reprobación, son indicios de una dificultad en la expresión afectiva y comunicativa familiar.

Cabe pensar que la relación entre una conducta desajustada - abuso de alcoholy las anteriores prácticas educativas, se explica a través de la sensación general de insatisfacción que queda en el hijo receptor de tales estrategias socializadoras. La convicción de no ser aceptado íntegra e incondicionalmente por sus progenitores, puede ser aparentemente disimulada por la integración en un grupo social que proporciona sentimientos de ubicación social y que satisface necesidades de afiliación mediante la elicitación de determinadas conductas rituales - consumos varios en el doble sentido de ingesta y dispendio- que suponen la imitación de comportamientos tácitamente aceptados como normativos del grupo. Si a esto añadimos la percepción de normalidad que acompaña al consumo social de alcohol, que el adolescente ha adquirido a través del modelado de la conducta de los adultos, el riesgo de convertirse en un consumidor abusivo se verá aumentado.

No obstante todo lo anterior, no podemos afirmar que el consumo de alcohol entre los adolescentes está ligado «únicamente» al consumo familiar y a las estrategias paternas de socialización. También las influencias derivadas del consumo en el grupo de iguales son un factor de riesgo a tomar en consideración $^{30}$. El inicio del hábito social de consumo de bebidas alcohólicas acontece, ciertamente, durante la adolescencia dentro del grupo de iguales; sin embargo, hemos visto que las variables derivadas del sistema familiar van a ser fundamentales para explicar dicha conducta, como ahora pretendemos justificar.

La conciencia de uso acrítico de una droga institucionalizada como el alcohol, se adquiere en interacción con los progenitores. Posteriormente, el adolescente verificará las «ventajas» sociales del uso de estas sustan- cias, cuando desarrolle su propia conducta social, en intcracción con su grupo de iguales. Pero las actitudes permisivas hacia el alcohol con que el muchacho llega a su propio grupo, no son en absoluto independientes de aquello que ha adquirido en su contexto familiar, sino que este contexto socializador puede «enseñar» a través de las conductas cotidianas de sus miembros qué conductas son normativas y por tanto trasladables a los ámbitos de relación extrafamiliares en los que el adolescente se integrará. Además, el sistema peculiar de relaciones que cada adolescente ha establecido con sus padres a lo largo de todo su proceso de socialización, es una variable decisiva para definir otros factores de profundidad más inmediata, como, entre otros, el autoconcepto del jóven, sus estilos de relación social, así como diversas variables de personalidad - locus de control, vulnerabilidad a la presión grupal, tolerancia a la frustración, estabilidad emocional, etc.—31,32. También será en el contexto relacional del grupo donde los jóvenes pongan en juego las anteriores características y, en función de la menor o mayor posibilidad de ajuste social y personal que éstas le permitan, así será la probabilidad de que inicie o no el consumo social de sustancias, o de que verifique o no un consumo abusivo de las mismas.

En definitiva, el consumo de bebidas alcohólicas no es una conducta que se dé en los adolescentes de manera aislada sin relación alguna con lo que ocurre en sus contextos sociales inmediatos, especialmente en el familiar; es, al contrario, un comportamiento cargado de significado social que refleja tanto la vulnerabilidad a la presión grupal, como las expectativas de mejor integración grupal a través de una conducta socialmente normativa, variables ambas que encuentran su origen en los procesos de socialización familiar.

Aquí es donde se pone de manifiesto el papel decisivo de la familia en las acciones preventivas. La eficacia de las estrategias educativas de la prevención primaria, es de- 
cir, de la anticipación de los factores de riesgo, supone la acción directa y responsable de los padres como agentes activos, pues ellos son quienes, en ocasiones, los propician sin ser conscientes. Esto supone el conocimiento por parte de los padres de los cambios biológicos, psicológicos y sociales que tienen lugar durante la adolescencia, la comprensión de los efectos que su propia conducta tendrá sobre las emociones, pensamientos y acciones de sus hijos, y la identificación de los factores de riesgo de las conductas desajustadas que se pretenden evitar. Acciones preventivas y de educación para la salud desarrolladas en este sentido, las encontramos propuestas por la Fundación de Ayuda contra la Drogadicción ${ }^{33}$, a través de programas de formación para padres, que suponen abordar la prevención te- niendo como punto de mira no tanto el propio adolescente, cuanto las relaciones que éste establece con los demás miembros de su entorno familiar, especialmente con sus progenitores.

Como conclusión general, apuntamos que las relaciones encontradas entre el consumo alcohólico por parte de los adolescentes, y las dos variables derivadas del contexto familiar que hemos estudiado, destacan la necesidad de implicar a los padres en los procesos preventivos, a fin de crear un ambiente familiar positivo y además ofrecer a los hijos un modelado racional y controlado en el uso familiar de bebidas alcohólicas. La prevención debería incluir a los padres como un agente central de la intervención. 
ANEXO

ESCALA INFLUCOL-92

\begin{tabular}{|c|c|c|c|}
\hline & Padre & Madre & Hermanos \\
\hline Vino & 1234 & 1234 & 1234 \\
\hline Cerveza & 1234 & 1234 & 1234 \\
\hline Carajillos & 1234 & 1234 & 1234 \\
\hline Combinados & 1234 & 1234 & 1234 \\
\hline Licores solos & 1234 & 1234 & 1234 \\
\hline Champañ & 1234 & 1234 & 1234 \\
\hline Vermut & 1234 & 1234 & 1234 \\
\hline
\end{tabular}

\section{ESCALA EMBU 89}

1. ¿Tienes la impresión de que tus padres ponen impedimentos a todo lo que haces?

2. ¿Te han demostrado con palabras y gestos que te quieren?

3. ¿Eres mimado por tus padres en comparación con tu(s) hermano(s)?

4. ¿Te sientes querido por tus padres?

5. ¿Dejan tus padres de dirigirte la palabra durante mucho tiempo si haces algo que les molesta?

6. ¿Te castigan tus padres incluso por cometer pequeñas faltas?

7. ¿Tratan tus padres de influirte para que seas una persona importante?

8. ¿Te sientes decepcionado en alguna ocasión porque tus padres no te conceden algo que tú deseas conseguir?

9. ¿Crees que tu padre o tu madre desean que tú seas diferente en algún aspecto?

10. ¿Te permiten tener cosas que no pueden tener tus hermanos?

11. ¿Piensas que tus padres te castigan merecidamente?

12. ¿Crees que tu padre o tu madre son demasiado severos contigo?

13. Si tú haces una trastada. ¿podrías remediar la situación pidiendo perdón a tus padres?

14. ¿Quieren siempre tus padres decidir cómo debes vestirte o qué aspecto debes tener?

15. ¿Te mienten tus padres? 
16. ¿Tienes la sensación de que tus padres te quieren menos que a tus hermanos?

17. ¿Te tratan tus padres injustamente en comparación a como tratan a tus hermanos?

18. ¿Tus padres te prohiben hacer cosas que otros chicos de tu edad pueden hacer, por miedo a que te suceda algo?

19. ¿Te riñen o te pegan tus padres en presencia de otras personas?

20. ¿Se preocupan tus padres de saber qué haces cuando no estás en casa?

21. Si las cosas te van mal, ¿tienes la sensación de que tus padres tratan de comprenderte y animarte?

22. ¿Se preocupan excesivamente tus padres por tu salud?

23. ¿Te imponen más castigos corporales de los que mereces?

24. ¿Se enfadan tus padres si no ayudas en las labores de la casa tanto como ellos desean?

25. Si a tus padres les parece mal lo que haces, ¿se entristecen hasta el punto de que te sientes culpable por lo que has hecho?

26. ¿Te permiten tus padres tener las mismas cosas que tus amigos?

27. ¿Tienes la sensación de que es difícil comunicarse con tus padres?

28. ¿Cuentan tus padres algo que tú has dicho o hecho, delante de otras personas, de forma que tú te sientas avergonzado?

29. ¿Sientes que tus padres te quieren menos que al resto de tus hermanos?

30. ¿Ocurre que tus padres no quieren concederte cosas que tú realmente necesitas?

31. ¿Muestran tus padres interés en que saques buenas notas?

32. ¿Sientes que tus padres te ayudan cuando te enfrentas a una tarea difícil?

33. ¿Te sientes tratado como la «oveja negra» de la familia?

34. ¿Desean tus padres que te parezcas a alguna otra persona?

35. Ocurre que tus padres te digan: «Tú que ya eres tan mayor no deberías comportarte de esa forma»?

36. ¿Critican tus padres a tus amigos(as) más íntimos(as)?

37. Cuando tus padres están tristes, ¿tienes la impresión de que ellos piensan que tú eres el causante de su estado?

38. ¿Intentan tus padres estimularte para que seas el mejor?

39. ¿Te demuestran tus padres que están satisfechos contigo? 
40. ¿Tienes la sensación de que tus padres confían en ti de tal forma que te permiten actuar bajo tu propia responsabilidad?

41. ¿Crees que tus padres respetan tus opiniones?

42. Si tú tienes pequeños secretos, ¿quieren tus padres que hables de estos secretos con ellos?

43. ¿Tienes la sensación de que tus padres quieren estar a tu lado?

44. ¿Crees que tus padres son algo tacaños y cascarrabias contigo?

45. ¿Utilizan tus padres expresiones como: «Si haces eso, voy a ponerme muy triste»?

46. Al volver a casa, ¿tienes que dar explicaciones a tus padres de lo que has estado haciendo?

47. ¿Crees que tus padres intentan que tu adolescencia sea estimulante, interesante y atractiva (por ejemplo, dándote a leer buenos libros, animándote a salir de excursión, etc.)?

48. ¿Tus padres alaban tu comportamiento?

49. ¿Emplean tus padres expresiones como ésta: «Así nos agradeces todo lo que nos hemos esforzado por ti y todos los sacrificios que hemos hecho por tu bien»?

50. ¿Ocurre que tus padres no e dejan tener cosas que necesitas, diciéndote que puedes convertirte en un chico mimado?

51. ¿Has llegado a sentir remordimiento (culpa) por comportarte de un modo que no sea del agrado de tus padres?

52. ¿Crees que tus padres tienen grandes esperanzas de que saques buenas notas, seas un buen deportista, etc.?

53. ¿Ignoran tus padres el que seas descuidado o tengas un comportamiento parecido?

54. Si te encuentras triste, ¿puedes buscar ayuda y comprensión en tus padres?

55. ¿Te castigan tus padres sin que tú hayas hecho nada mal?

56. ¿Te dejan tus padres hacer las mismas cosas que pueden hacer tus amigos?

57. ¿Te dicen tus padres que no están de acuerdo con tu forma de comportarte en casa?

58. ¿Te obligan tus padres a comer más de lo que puedes?

59. ¿Te critican tus padres o te dicen que eres vago o inútil delante de otras personas?

60. ¿Se interesan tus padres por el tipo de amigos con quienes vas?

61. De tus hermanos, ¿es a ti a quien tus padres echan la culpa de cuanto pasa? 
62. ¿Te aceptan tus padres tal como eres?

63. ¿Son bruscos tus padres contigo?

64. ¿Te castigan tus padres con dureza, incluso por cosas que no tienen importancia?

65. ¿Te pegan tus padres sin motivo?

66. ¿Deseas que tus padres se preocupen menos de las cosas que haces?

67. ¿Participan tus padres activamente en tus diversiones y en tus hobbys?

68. ¿Te pegan tus padres?

69. ¿Puedes ir donde quieres sin que tus padres se preocupen demasiado por ello?

70. ¿Te ponen tus padres limitaciones estrictas a lo que puedes y no puedes hacer, y te obligan a respetarlas rigurosamente?

71. ¿Te tratan tus padres de manera que puedas sentirte avergonzado?

72. ¿Les permiten tus padres a tus hermanos tener cosas que a ti no te dejan tener?

73. ¿Crees que es exagerado el miedo que tienen tus padres de que a ti te pase algo?

74. ¿Tienes la sensación de que hay cariño y ternura entre tú y tus padres?

75. ¿Respetan tus padres el hecho de que tú tengas opiniones diferentes a las suyas?

76. ¿Recuerdas si tus padres han estado enfadados o amargados contigo sin que te dijeran el por qué?

77. ¿Te han mandado tus padres a la cama sin cenar?

78. ¿Tienes la impresión de que tus padres se sienten orgullosos de ti cuando consigues lo que te has propuesto?

79. ¿Muestran tus padres predilección por ti en comparación con tus hermanos?

80. ¿Echan tus padres las culpas a tus hermanos aunque seas tú el responsable de lo que ha ocurrido?

81. ¿Te manifiestan tus padres que están satisfechos contigo mediante expresiones físicas cariñosas, como darte palmadas en la espalda? 
ESCALA HABICOL-92

\begin{tabular}{|lcccc|}
\hline Vino (vasos) & 0 & $1-3$ & $4-6$ & +6 \\
\hline Cerveza: & & & & \\
(cañas) & 0 & $1-3$ & $4-6$ & +6 \\
(botellín o vaso grande) & 0 & $1-2$ & $3-4$ & +4 \\
(jarras individuales) & 0 & 1 & 2 & +2 \\
Carajillos & 0 & 1 & 2 & +2 \\
Combinados (vasos) & 0 & $1-2$ & $3-4$ & +4 \\
Licores (copas) & 0 & $1-2$ & $3-4$ & +4 \\
Champañ (copas) & 0 & $1-2$ & $3-4$ & +4 \\
Vermut (vasos) & 0 & $1-2$ & $3-4$ & +4 \\
\hline «Litrona» entre varios: & & & & \\
entre dos & & & & +4 \\
entre tres & 0 & $1-2$ & $3-4$ & +4 \\
entre cuatro & 0 & $1-2$ & $3-4$ & +4 \\
entre más de cuatro & 0 & $1-2$ & $3-4$ & +4 \\
\hline «Cubalitro" entre varios: & 0 & $1-2$ & $3-4$ & +4 \\
entre dos & & & & \\
entre tres & 0 & $1-2$ & $3-4$ & +4 \\
entre cuatro & 0 & $1-2$ & $3-4$ & +4 \\
entre más de cuatro & 0 & $1-2$ & $3-4$ & +4 \\
\hline
\end{tabular}

\section{BIBLIOGRAFÍA}

1. Silverman, S.M.. Prevención del uso indebido de drogas: La comunidad en acción. Nueva York: O.N.U; 1991.

2. Pons, J. y Buelga, S.. Familia y conductas desviadas: El consumo de alcohol. En: G. Musitu y P. Allat (Eds.) Psicosociología de la familia. Valencia: Albatros; 1994.

3. Musitu, G. La violencia familiar: Un análisis desde el Interaccionismo Simbólico. Universitas Tarraconensis; 1986. p. 1-2.

4. García, A. y Ezquiaga, E. Estudio descriptivo de una muestra de 433 drogodependientes que acudieron a un centro de salud mental. Adicciones 1991: 167-180.

5. Jiménez, J.L. y Revuelta, A. La familia del toxicómano: Un estudio comparativo. Adicciones 1991: 133-140.

6. Salvador, T. Políticas europeas de prevención del alcohol y otras drogodependencias. En: Alcohol y adolescencia: Hacia una educación preventiva. Madrid: CCS; 1994.
7. Cloninger, C.R., Bohman, M. y Sigvardson, S. Inheritance of alcohol abuse: Cross-fostering analysis of adopted men. Arch Gen Psychiatry 1981: 861-868.

8. Goodwin, D.W. The role of genetics in the expression of alcoholism: Overview. En: M. Galanter (Ed.), Recent developments in alcoholism. Nueva York: Plenum Press; 1983.

9. Midanik, L. Familial alcoholism and problem drinking in a national drinking pactices survey. Addictive behaviors 1983: 133-141.

10. Pons, J. Autoconcepto, comunicación familiar y consumo de drogas en alumnos de Segundo Ciclo de E.G.B. [Tesis de Licenciatura]. Valencia: Universitat de València; 1989.

11. Protinsky, H. y Shilts, L. Adolescent substance use and family cohesion. Family Therapy 1990: 173175.

12. Pardeck, J.T. A multiple regression analysis of family factors affecting the potential for alcoholism in college students. Family Therapy 1991: 115-121.

13. Noller, P. y Callan, V. The adolescent in the family. Londres: Routledge; 1991. 
14. Fontaine, A.M., Campos, B.P. y Musitu, G. Percepção das interacções familiares e conceito de si próprio na adolescência. Cadernos de Consulta Psicológica 1992: 69-78.

15. Lackovic-Grgin, K. y Dekovic, M. The contribution of significant others to adolescents' self-esteem. Adolescence 1990:839-846.

16. Peinado, A., Pereña, F y Portero, P. La cultura del alcohol entre los jóvenes de la Comunidad de Madrid. Madrid: Comunidad de Madrid; 1993.

17. Parra, J. Los adolescentes y su cultura del alcohol y de la noche. En: Alcohol y adolescencia: Hacia una educación preventiva. Madrid: CCS; 1994.

18. Perris, C., Jacobson, L., Lindström, H., Von Knorring, L. y Perris, H. Development of a new inventory for assessing memories of parental rearing behavior. Acta Psychiatr Scand 1980: 265-274.

19. Arrindell, W., Perris, C., Perris, H., Eisemann, M., Perris, H., Van der Ende, J., Ross, M., Benjaminsen, S., Gaszner, P. y Del Vecchio, M. Cross-national generalizability of patterns of parental rearing behavior: Invariance of EMBU dimensional representations of healthy subjects from Australia, Denmark, Hungary, Italy and The Netherlands. Personality and Individual Differences 1986: 103-112.

20. Musitu, G., Molpeceres, M.A., García, F. y Lila, M.S. Dimensiones percibidas de la socialización: Una contrastación transcultural de la estructura factorial del cuestionario EMBU (Perris et al., 1980). En: G. Musitu, M. Gutiérrez y J. Pons (Eds.), Intervención comunitaria. Valencia: Set i Set Edicions; 1994.

21. Estarelles, R. Clima familiar y autoconcepto en la adolescencia. [Tesis Doctoral]. Valencia: Universital de València; 1986.

22. Gutiérrez, M. Interacción familiar, autoconcepto y conducta prosocial. [Tesis Doctoral]. Valencia: Universitat de València; 1989.

23. Herrero, J., Musitu, G., García, F. y Gomis, M.J. Las prácticas educativas de los padres en la ado- lescencia. Actas del III Congreso Nacional de Psicología Social 1991: 352-361.

24. Cruz Roja Española. Estudio epidemiológico sobre el consumo de alcohol en adolescentes en La Rioja y Cádiz. Madrid: Intercampo; 1985.

25. Cárdenas, C. El consumo de bebidas alcohólicas en la adolescencia: Condiciones contribuyentes. Drogalcohol 1986: 58-65.

26. Martínez, M.J., Aguinaga, M. y Varo, J.R. El consumo de alcohol por los jóvenes y su percepción del uso de alcohol por los padres. En: Avances en drogodependencias. Córdoba: Diputación de Córdoba; 1992.

27. Budd, R.J., Eiser, J., Morgan, M. y Gammage, P. The personal characteristics and life-style of the young drinker: The results of a survey of British adolescents. Drug and Alcohol Dependence 1985: 145-157.

28. Elzo, J., Amatria, M., González de Audicana, M. Echeburua, E. y Ayestarán, S. Drogas y escuela III. San Sebastián: Escuela Universitaria de Trabajo Social; 1987.

29. Berjano, E. Análisis psicosociológico del consumo de drogas en el contexto escolar: Mecanismos de prevención. [Tesis Doctoral]. Valencia: Universitat de València; 1988.

30. Fromme, K. y Ruela, A. Mediators and moderators of young adults drinking. Addiction 1994: 63-71.

31. Funes, J. La nueva delincuencia juvenil. Barcelona: Paidós; 1984.

32. Lila, M.S., Musitu, G. y Molpeceres, M.A. Familia y autoconcepto. En: G. Musitu y P. Allat (Eds.), Psicosociología de la familia. Valencia: Albatros; 1994.

33. Fundación de Ayuda contra la Drogadicción. Curso de prevención de drogas. Manual y fichas para los padres. Las Palmas de Gran Canaria: FADFundación Ecca; 1991. 